\title{
Enhanced Photosynthesis of Carotenoids in Microalgae Driven by Light-Harvesting Gold Nanoparticles
}

Xiaojie Li, ${ }^{1,2,3}$ Han Sun, ${ }^{4}$ Xuemei Mao, ${ }^{1,3}$ Yongmin Lao, ${ }^{1,3}$ Feng Chen*1, 3

${ }^{1}$ Shenzhen Key Laboratory of Marine Microbiome Engineering, Institute for Advanced Study, Shenzhen University, Nanshan District Shenzhen, Guangdong, 518060, China

2 Key Laboratory of Optoelectronic Devices and Systems of Ministry of Education and Guangdong Province, College of Optoelectronic Engineering, Shenzhen University, Shenzhen, 518060, China

${ }^{3}$ Institute for Innovative Development of Food Industry, Shenzhen University, Nanshan District Shenzhen, Guangdong, 518060, China

${ }^{4}$ Institute for Food \& Bioresource Engineering, College of Engineering, Peking University, Yiheyuan Road Haidian District, Beijing, 100871, China

Corresponding author: * (F Chen) E-mail: sfchen@szu.edu.cn; Phone: +86-0755-26531167

\section{Table of Contents}

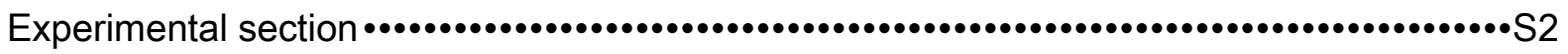

Figure S1. TEM images of as-synthesized AUNPs

Figure S2. Size distributions of as-synthesized AuNPs •................................S4

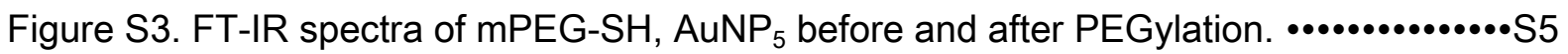

Figure S4. Colloidal stability of as-synthesized AuNPs in BG11 medium •.................S6

Figure S5. Size changes of AuNPs in BG11 medium •..................................S7

Figure S6. TEM Images of AuNPs after incubation in BG11medium •......................S8

Figure S7. Biomass concentration of $C$. zofingiensis cultured with AuNPs $\cdot \cdots . . . . . . . . . . . . . . S 9$

Figure S8. Light microscopy images of $C$. zofingiensis cultured with AuNPs

Figure S9. Photosynthetic yield of $C$. zofingiensis treated with different AuNPs............S11 


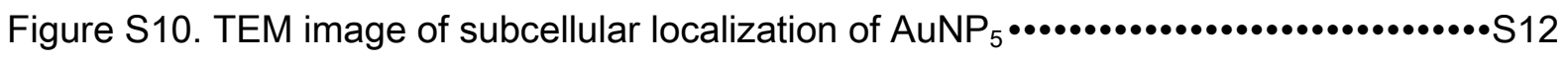
Figure S11. Carotenoid yield of $C$. zofingiensis-AuNP 5 biohybrid system on Day3 and Day 5

Figure S12. Carotenoid yield of C. zofingiensis-AuNP 5 biohybrid system under light and dark

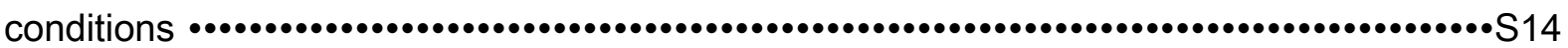

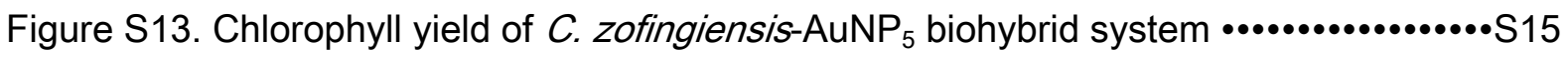

Figure S14. Biomass concentration of $C$. zofingiensis-AuNP ${ }_{5}$ biohybrid system $\cdots \cdots \cdots \cdots . . . S 16$

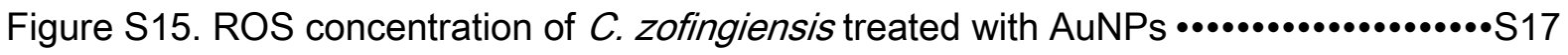

Table S1. The comparisons of carotenoid yield among different strategies Reference S19

Number of pages: 19

Number of figures: 15

Number of tables: 1 


\section{Experimental section}

Measurement of photosynthetic yield. The yield reflects the efficiency of the photochemical energy conversion process. ${ }^{1}$ After cultured with 40 ppm AuNPs for 3 days, algal cells were collected by centrifuge and diluted to the similar absorbance at $680 \mathrm{~nm}$. The photosynthetic yield of $C$. zofingiensis were measured by using PAMfluorometry (WATER-PAM, Walz, Germany) at the same light condition that used for microalgae growth ( $40 \mu \mathrm{mol} \mathrm{m} \mathrm{m}^{-2} \mathrm{~s}^{-1}$ PAR irradiation). 

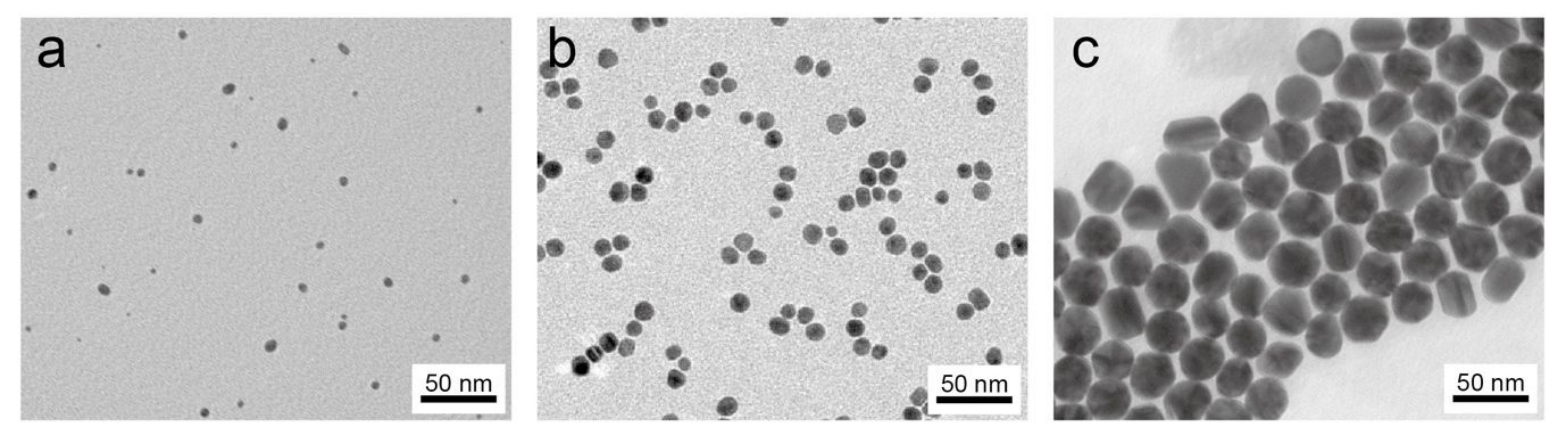

Figure S1. TEM images of as-synthesized (a) $\mathrm{AuNP}_{5}$, (b) $\mathrm{AuNP}_{15}$ and (c) $\mathrm{AuNP}_{30}$. 

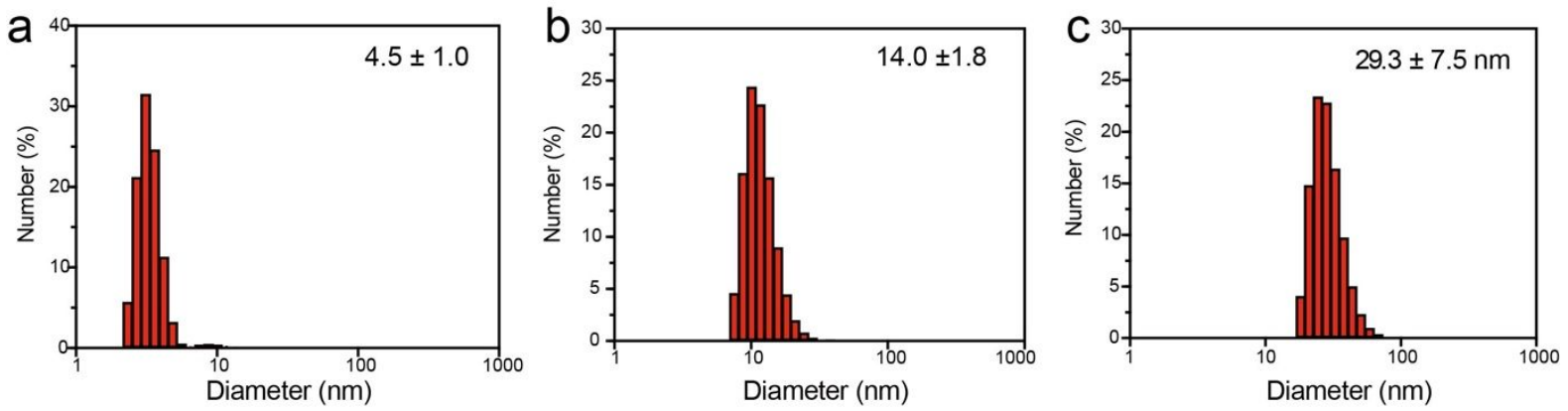

Figure S2. Size distributions of as-synthesized (a) $\mathrm{AuNP}_{5}$, (b) $\mathrm{AuNP}_{15}$ and (c) $\mathrm{AuNP}_{30}$. 


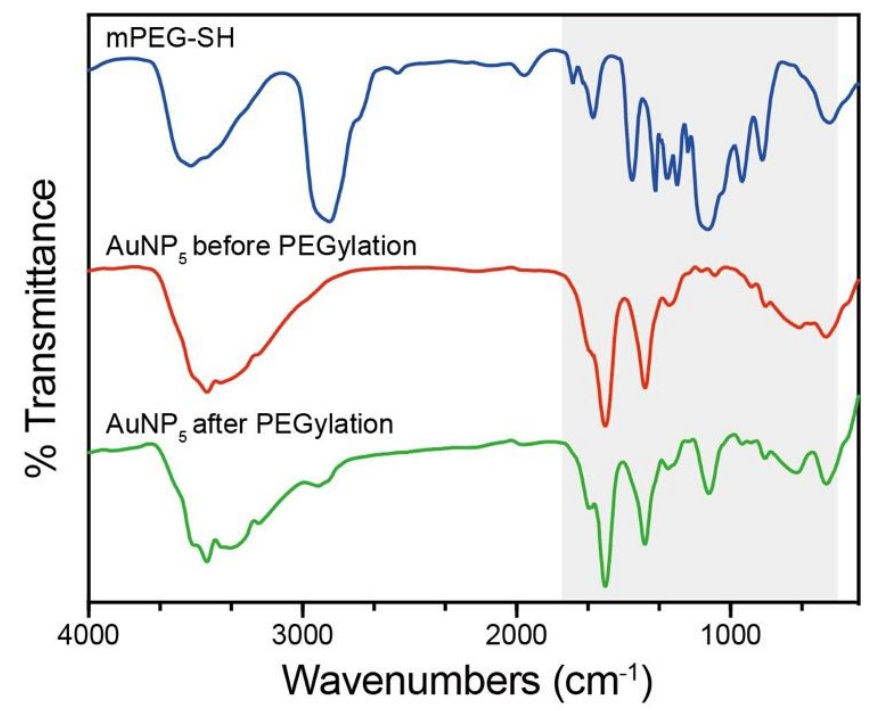

Figure S3. FT-IR spectra of mPEG-SH, $\mathrm{AuNP}_{5}$ before and after PEGylation. 


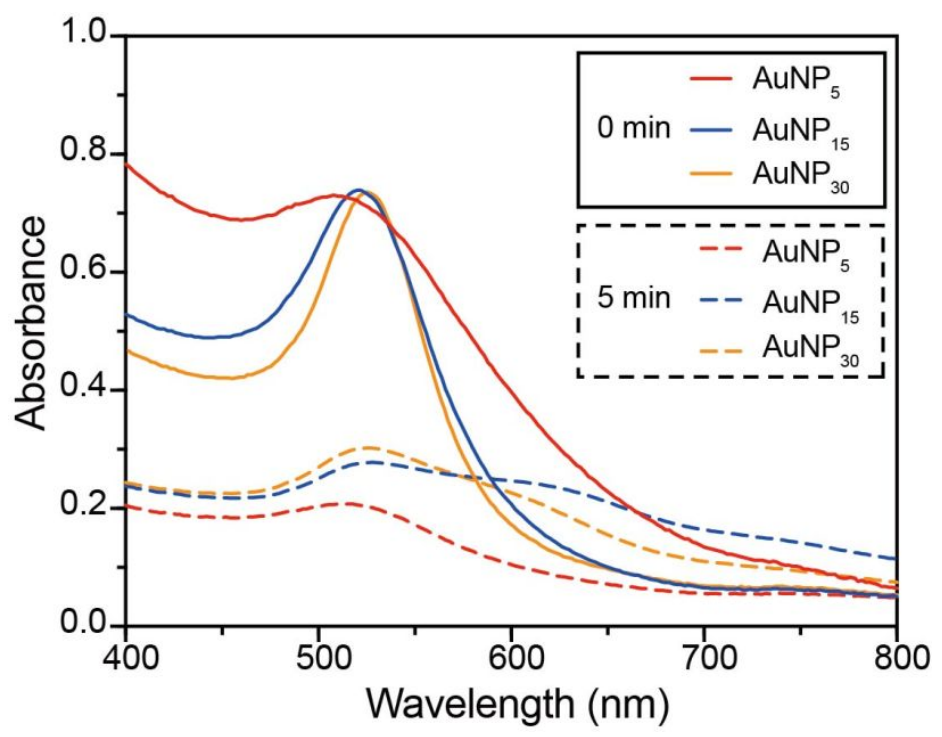

Figure S4. Colloidal stability of as-synthesized AuNPs in BG11 medium. The decreased intensity and red-shift of UV-Vis spectra indicated the aggregation of the nanoparticles. 


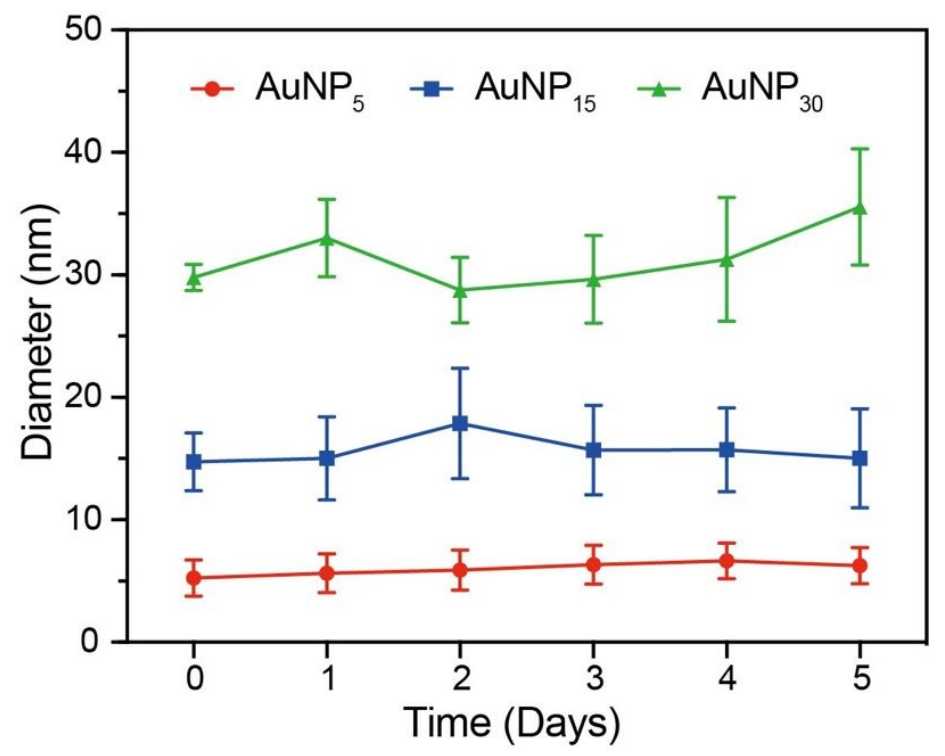

Figure S5. Size changes of AuNPs during 5-day incubation in BG11 medium. All points and error bars are shown as means $\pm \mathrm{SD}(\mathrm{n}=3)$. 

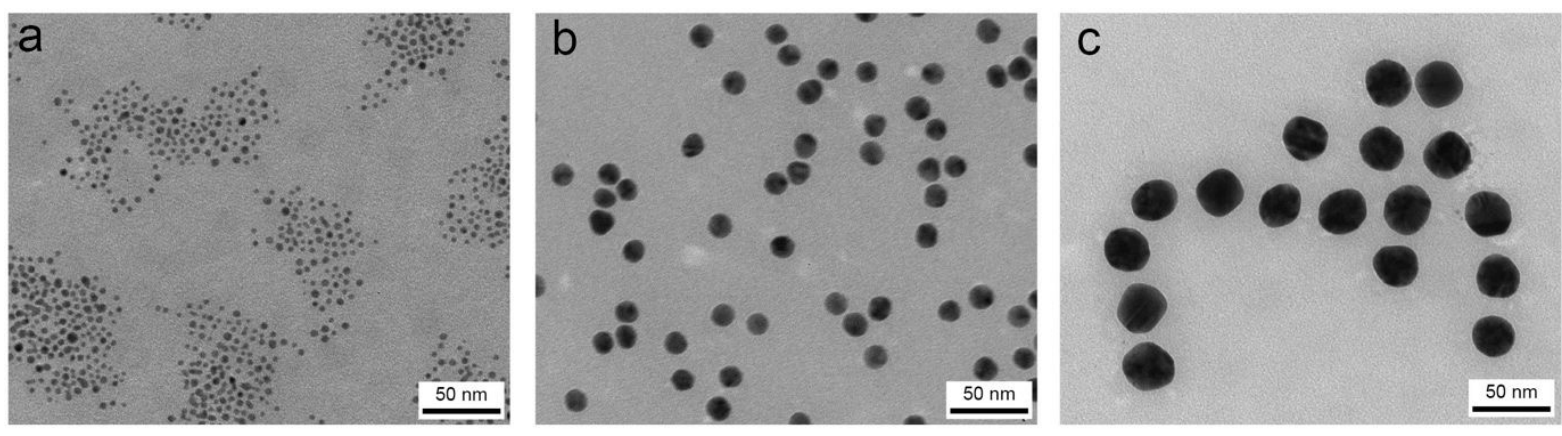

Figure S6. TEM Images of (a) $\mathrm{AuNP}_{5}$, (b) $\mathrm{AuNP}_{15}$ and (c) $\mathrm{AuNP}_{30}$ after incubation in BG11medium for 5 days. 

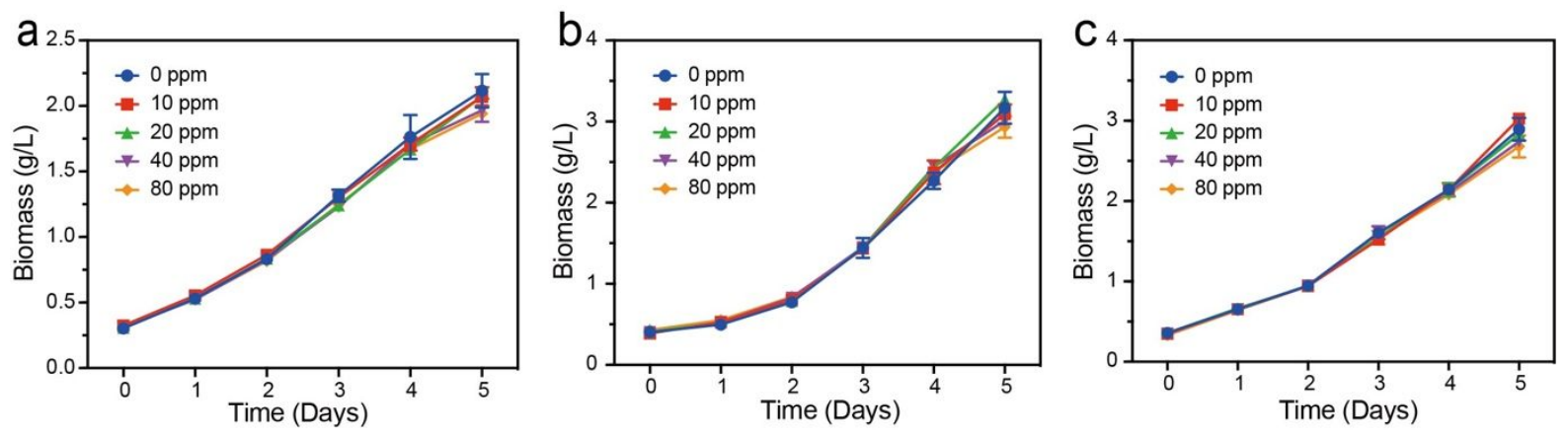

Figure S7. Biomass concentration of $C$. zofingiensis cultured with different concentration of

(a) $\mathrm{AuNP}_{5}$, (b) $\mathrm{AuNP}_{15}$ and (c) $\mathrm{AuNP}_{30}$ under light condition. All points and error bars are shown as means $\pm \mathrm{SD}(\mathrm{n}=3)$. 

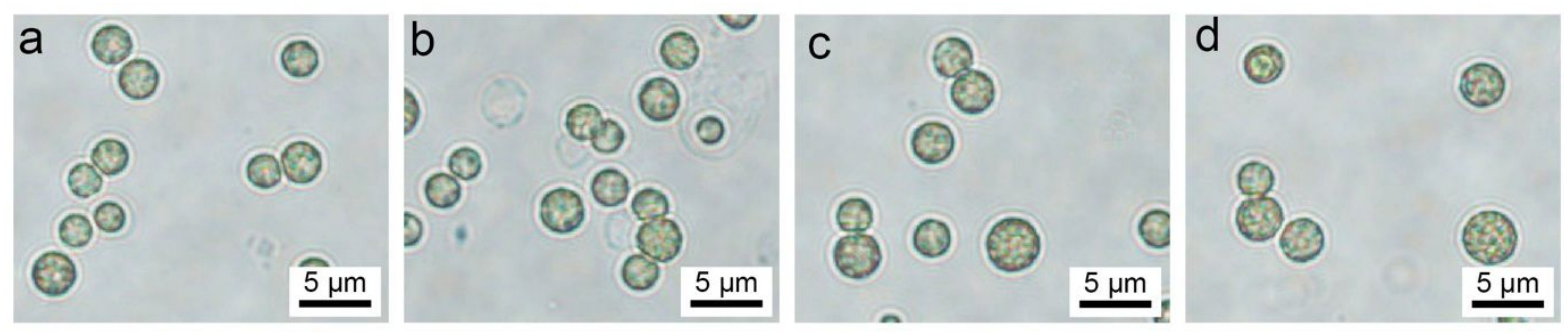

Figure S8. Light microscopy images of C. zofingiensis cultured with (a) 0 ppm AuNPs and 80 ppm (b) $\mathrm{AuNP}_{5}$, (c) $80 \mathrm{ppm} \mathrm{AuNP} 15$ and (d) $80 \mathrm{ppm} \mathrm{AuNP} 30$ under light condition for 5 days. 


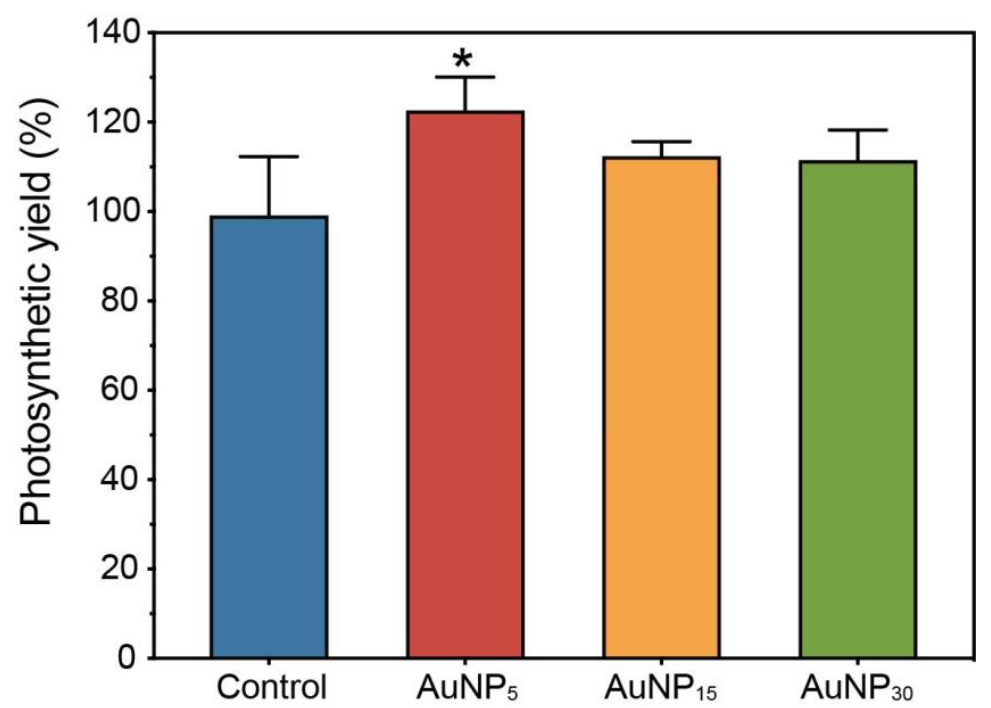

Figure S9. Photosynthetic yield (\%) of C. zofingiensis treated with different AuNPs under light condition. All points and error bars are shown as means $\pm \mathrm{SD}(\mathrm{n}=3)$. An asterisk indicates a significant difference between treatments $(* p<0.05)$. 

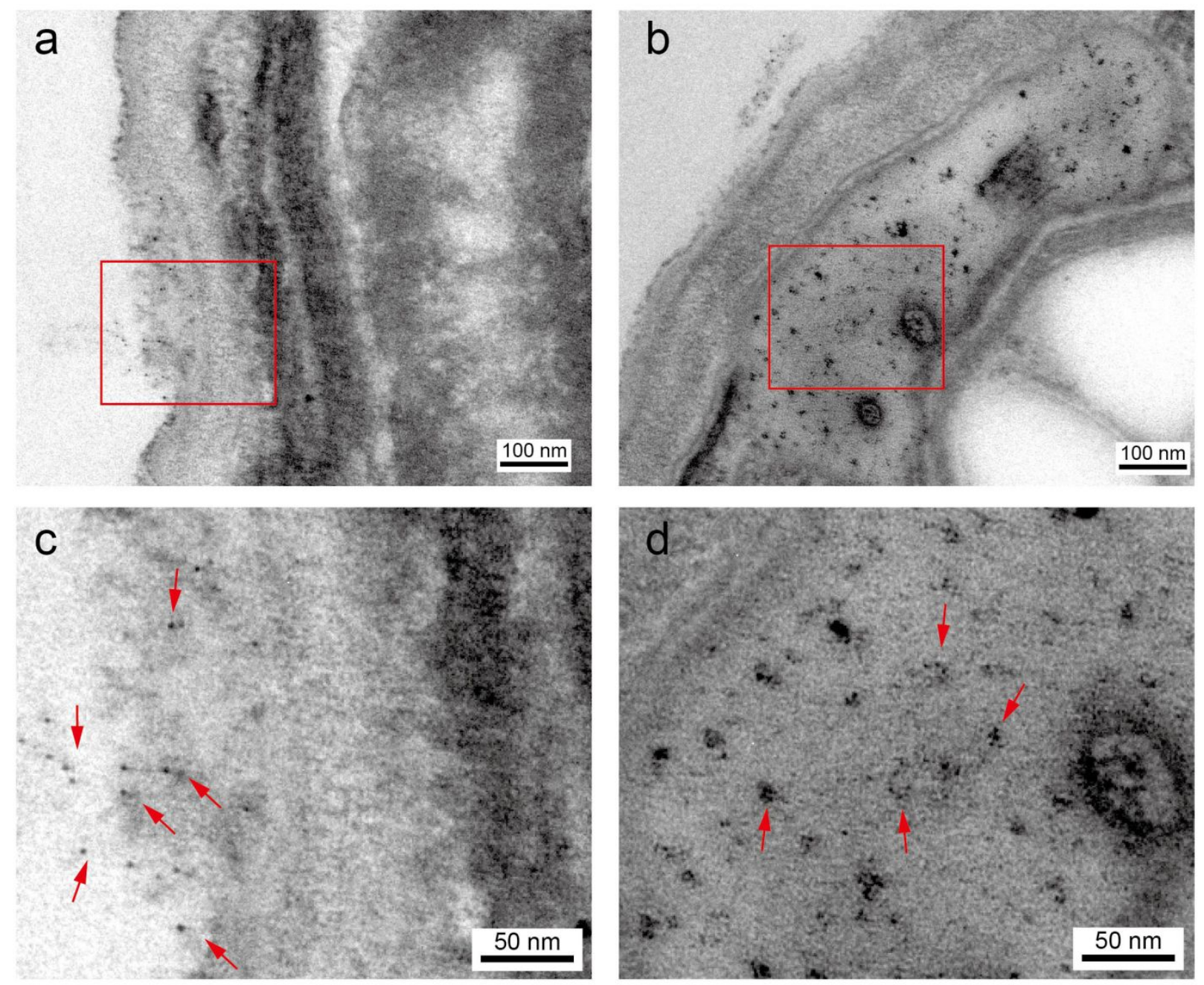

Figure S10. (a) and (b) are TEM images of $\mathrm{AuNP}_{5}$ in different locations of $C$. zofingiensis cells. (c) and (d) are the magnified images of the highlighted regions of (a) and (b), respectively. 


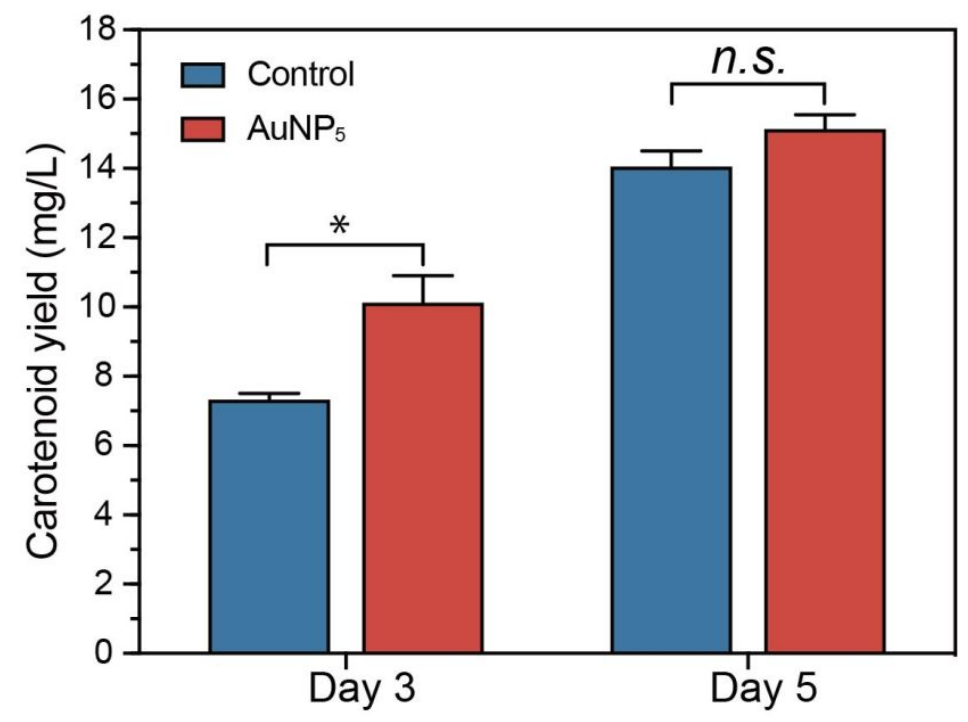

Figure S11. Carotenoid yield in C. zofingiensis-AuNP ${ }_{5}$ hybrid system on Day 3 and Day 5 under light condition. All points and error bars are shown as means $\pm \mathrm{SD}(\mathrm{n}=3)$. An asterisk indicates a significant difference between treatments $(* p<0.01)$; n.s., no significant difference. 


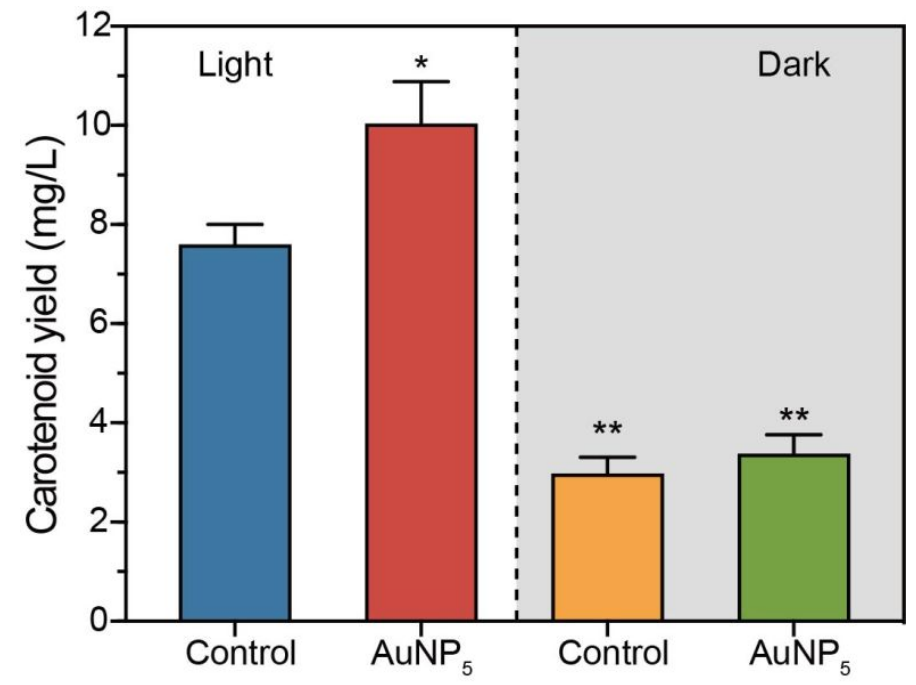

Figure S12. Carotenoid yield of C. zofingiensis-AuNP 5 biohybrid system under light and dark conditions. All points and error bars are shown as means $\pm \mathrm{SD}(\mathrm{n}=3)$. An asterisk indicates a significant difference between treatments $(* p<0.01$, ** $p<0.001)$; n.s., no significant difference. 


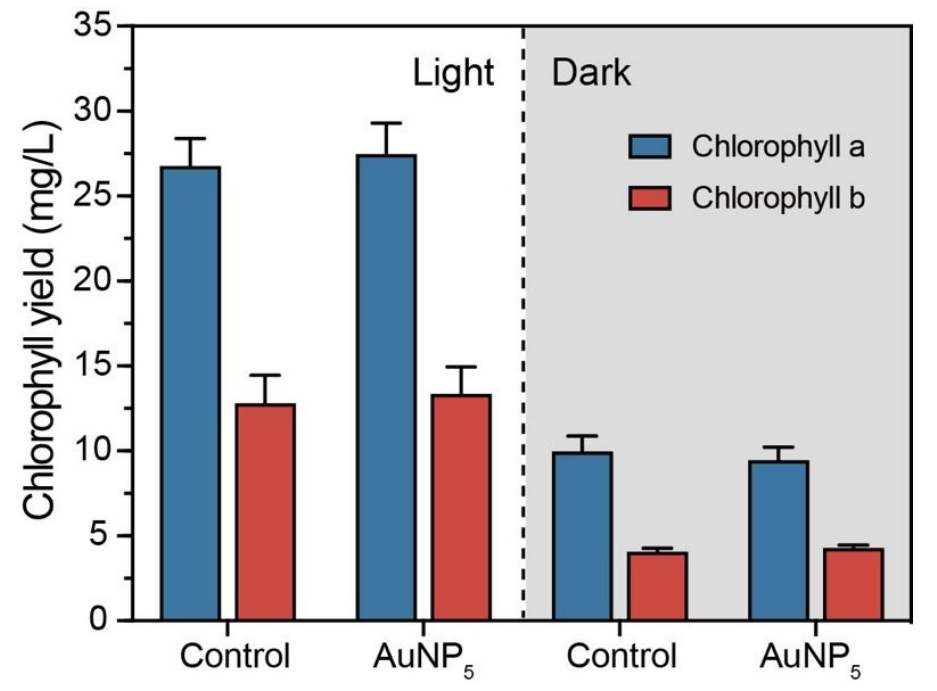

Figure S13. Chlorophyll yield of $C$. zofingiensis-AuNP ${ }_{5}$ biohybrid system in light and dark conditions. All points and error bars are shown as means $\pm \operatorname{SD}(n=3)$. 


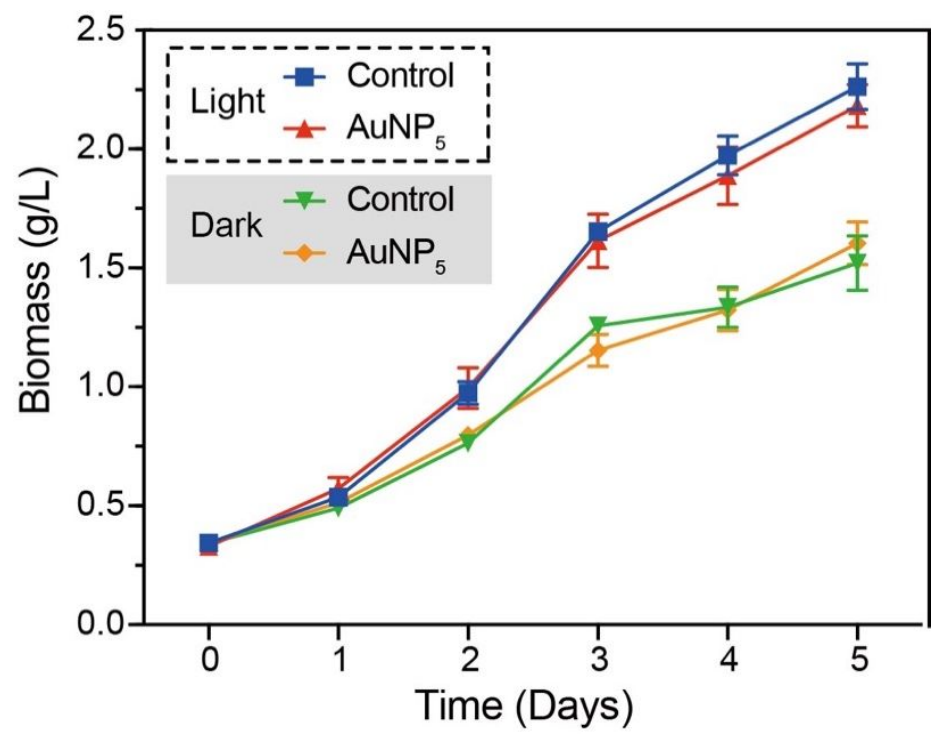

Figure S14. Biomass concentration of $C$. zofingiensis-AuNP ${ }_{5}$ biohybrid system under light and dark conditions. All points and error bars are shown as means $\pm \operatorname{SD}(n=3)$. 


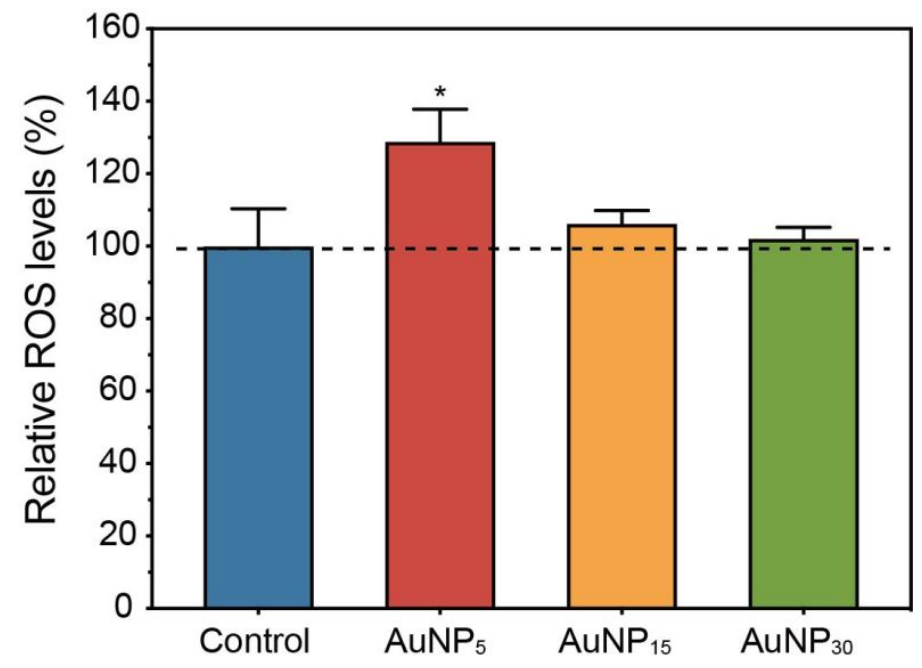

Figure S15. ROS concentration of $C$. zofingiensis treated with different AuNPs under light condition. All points and error bars are shown as means $\pm \mathrm{SD}(\mathrm{n}=3)$. An asterisk indicates a significant difference between treatments $(* p<0.01)$. 
Table S1. The comparisons of carotenoid yield among this study and others.

\begin{tabular}{|c|c|c|c|c|}
\hline Species & Culture method & $\begin{array}{c}\text { Carotenoid yield } \\
\text { increased (\%) }\end{array}$ & Remarks & Ref. \\
\hline C. zofingiensis & $\begin{array}{l}\text { Construction of } C \text {. } \\
\text { zofingiensis-AuNPs hybrid } \\
\text { systems under light condition } \\
\left(40 \mu \mathrm{mol} \mathrm{m}-2 \mathrm{~s}^{-1}\right)\end{array}$ & $42.7 \%$ & $\begin{array}{l}\text { 3-day cultivation; } \\
\text { No inhibition on biomass }\end{array}$ & $\begin{array}{l}\text { This } \\
\text { study }\end{array}$ \\
\hline C. zofingiensis & Phosphorus starvation & $27.6 \%$ & $\begin{array}{l}\text { 5-day cultivation } \\
\text { Decrease in biomass. }\end{array}$ & 2 \\
\hline C. zofingiensis & $\begin{array}{l}\text { Two-step cultivation with } \\
\text { nitrogen stravation and high } \\
\text { light }\left(350 \mu \mathrm{mol} \mathrm{m}^{-2} \mathrm{~s}^{-1}\right)\end{array}$ & Not available & 18-day cultivation & 3 \\
\hline C. reinhardtii & $\begin{array}{l}\text { Nitrogen starvation and high } \\
\text { light }\left(150 \mu \mathrm{mol} \mathrm{m}{ }^{-2} \mathrm{~s}^{-1}\right)\end{array}$ & $21 \%$ & $\begin{array}{l}\text { 4-day cultivation; } \\
\text { Decrease in Fv/Fm and } \\
\text { ETR }\end{array}$ & 4 \\
\hline C. zofingiensis & $\begin{array}{l}\text { Transformation of } \\
\text { microalgae to overexpress of } \\
P D S \text { gene and then cultured } \\
\text { with high light }\left(300 \mu \mathrm{mol} \mathrm{m}^{-}\right. \\
\left.{ }^{2} \mathrm{~S}^{-1}\right)\end{array}$ & $32.1 \%$ & 2-day cultivation & 5 \\
\hline
\end{tabular}




\section{REFERENCES}

1. Navarro, E.; Wagner, B.; Odzak, N.; Sigg, L.; Behra, R., Effects of Differently Coated Silver Nanoparticles on the Photosynthesis of Chlamydomonas reinhardtii. Environ Sci Techno/2015, 49(13), 8041-7, DOI 10.1021/acs.est.5b01089.

2. Mao, X.; Wu, T.; Sun, D.; Zhang, Z.; Chen, F., Differential responses of the green microalga Chlorella zofingiensis to the starvation of various nutrients for oil and astaxanthin production. Bioresource Technology 2018, 249, 791-798, DOI 10.1016/j.biortech.2017.10.090.

3. Zhang, Z.; Huang, J. J.; Sun, D.; Lee, Y.; Chen, F., Two-step cultivation for production of astaxanthin in Chlorella zofingiensis using a patented energy-free rotating floating photobioreactor (RFP). Bioresource Technology 2017, 224, 515-522, DOI 10.1016/j.biortech.2016.10.081.

4. Sun, H.; Mao, X.; Wu, T.; Ren, Y.; Chen, F.; Liu, B., Novel insight of carotenoid and lipid biosynthesis and their roles in storage carbon metabolism in Chlamydomonas reinhardtii. Bioresource Technology 2018, 263, 450-457, DOI 10.1016/j.biortech.2018.05.035. 
5. Liu, J.; Sun, Z.; Gerken, H.; Huang, J.; Jiang, Y.; Chen, F., Genetic engineering of the green alga Chlorella zofingiensis: a modified norflurazon-resistant phytoene desaturase gene as a dominant selectable marker. Appl Microbiol Biotechnol 2014, 98(11), 5069-79, DOI 10.1007/s00253-014-5593-y. 